\title{
Anomaly Cancellation with an Extra Gauge Boson
}

\author{
B. C. Allanach $\odot^{*}$ \\ DAMTP, University of Cambridge, Wilberforce Road, Cambridge CB3 OWA, United Kingdom \\ Ben Gripaios $\oplus^{\dagger}$ and Joseph Tooby-Smith $\oplus^{\ddagger}$ \\ Cavendish Laboratory, University of Cambridge, J.J. Thomson Avenue, Cambridge CB3 OHE, United Kingdom
}

(Received 11 June 2020; revised 12 July 2020; accepted 9 September 2020; published 14 October 2020)

\begin{abstract}
Many extensions of the standard model include an extra gauge boson, whose couplings to fermions are constrained by the requirement that anomalies cancel. We find a general solution to the resulting diophantine equations in the plausible case where the chiral fermion content is that of the standard model plus three right-handed neutrinos.
\end{abstract}

DOI: 10.1103/PhysRevLett.125.161601

Introduction.-Given the existence of a heavy, neutral gauge boson in the standard model (SM) of particle physics - the $Z$ boson-it is natural to ask whether there may be others. Such a $Z^{\prime}$, which corresponds to adding an additional $\mathfrak{t}(1)$ to the gauge Lie algebra $\mathfrak{g} \mathfrak{u}(3) \oplus \mathfrak{s} \mathfrak{u}(2) \oplus$ $\mathfrak{t}(1)$ of the SM, has featured in many models extending the SM [1] and has been the target of myriad experimental searches [3]. The couplings of the $Z^{\prime}$ to fermionic matter (such as the quarks and leptons of the SM, as well as righthanded neutrinos) cannot be arbitrarily chosen; just as for the hypercharge gauge boson, we expect first that they should be commensurate (corresponding to the expectation that the gauge group is compact, although we note that we can solve for the noncommensurate case, which we discuss briefly in the closing remarks) and second that anomalies (which would spoil the consistency of theory at the quantum level [4]) should cancel [6]. The former implies that the fermion charges under the extra $\mathfrak{u}(1)$ can be taken to be integers (any overall real factor can be absorbed into the gauge coupling) and the latter implies that they solve the homogeneous polynomial equations

$$
\begin{aligned}
& 0=\sum_{i=1}^{3}\left(6 Q_{i}+3 U_{i}+3 D_{i}+2 L_{i}+E_{i}+N_{i}\right), \\
& 0=\sum_{i=1}^{3}\left(3 Q_{i}+L_{i}\right),
\end{aligned}
$$

Published by the American Physical Society under the terms of the Creative Commons Attribution 4.0 International license. Further distribution of this work must maintain attribution to the author(s) and the published article's title, journal citation, and DOI. Funded by SCOAP ${ }^{3}$.

$$
\begin{aligned}
& 0=\sum_{i=1}^{3}\left(2 Q_{i}+U_{i}+D_{i}\right), \\
& 0=\sum_{i=1}^{3}\left(Q_{i}+8 U_{i}+2 D_{i}+3 L_{i}+6 E_{i}\right), \\
& 0=\sum_{i=1}^{3}\left(Q_{i}^{2}-2 U_{i}^{2}+D_{i}^{2}-L_{i}^{2}+E_{i}^{2}\right), \\
& 0=\sum_{i=1}^{3}\left(6 Q_{i}^{3}+3 U_{i}^{3}+3 D_{i}^{3}+2 L_{i}^{3}+E_{i}^{3}+N_{i}^{3}\right) .
\end{aligned}
$$

Here we have assumed that the chiral [7] fermions (all of which, via charge conjugation, may be taken to have the same chirality) consist of the just three SM families of quarks and leptons, together with three right-handed neutrinos, whose charges we label by $Q_{i}, U_{i}, D_{i}, L_{i}, E_{i}$, $N_{i}$, respectively, with $i \in\{1,2,3\}$. We consider this to be the most plausible scenario, on the grounds of both aesthetics and observation (e.g., the fit to neutrino oscillation data), and so we postpone comment on other possibilities to the closing remarks.

Finding any solutions to diophantine equations (or even establishing their existence or otherwise) is, in general, a notoriously difficult problem in number theory (very roughly, the state of the art is a single cubic in three unknowns). Surprisingly, we will see that one can, in fact, find all solutions to (1a)-(1f). These solutions inform models where the rank of the SM is increased, since the extra $\mathfrak{t}(1)$ may be a subalgebra of some larger additional gauge extension, as well as future phenomenological $Z^{\prime}$ studies.

Sketch of the solution.-The keys to solving (1a)-(1f) are twofold. The first is to convert it to a problem in geometry by observing that one can equivalently seek rational 
solutions (since any integer solution trivially defines a rational solution and since, by clearing denominators, every rational solution defines an integer solution). The rational numbers form a field, allowing one to carry out division and hence various basic geometrical constructions. The 18 charges appearing in (1a)-(1f) then form coordinates for the affine space $\mathbb{Q}^{18}$. In fact, given that scaling all charges by a common multiple leads to the same physics (as we have remarked, the scaling can be absorbed in a redefinition of the gauge coupling), it is convenient to consider not the charges themselves, but the equivalence classes under such a scaling, which define the projective space $P \mathbb{Q}^{17}$ (whose points we sometimes call rational points for emphasis). The homogeneous polynomials (1a)-(1f) define a projective variety in $P \mathbb{Q}^{17}$ whose points, which we call rational solutions, we seek.

The second key to solving the problem is that it is easy enough to find some rational solutions, (e.g., by means of a numerical scan [8]); three such points, $A, B$, and $C$, are defined in Table I. These can be used as the starting point for geometric constructions. To give an example, consider just the quadratic (1e) and suppose we know one rational point on the quadratic, $C$ say. Ignoring degenerate cases for now, a line $L$ through $C$ intersects the quadratic at 1 other rational point $R$ and moreover every rational point on the quadratic (indeed every point in the ambient space) lies on a line through $C$. Thus, by parametrizing all such lines, all rational points on the quadratic may be found [9].

To solve the full set of Eqs. (1a)-(1f) will require a more elaborate construction, as follows. First, we note that the four linear equations (1a)-(1d) simply define a projective subspace of $P \mathbb{Q}^{17}$ isomorphic to $P \mathbb{Q}^{13}$, to which we restrict our attention in what follows. Second, we exploit the fact that $B$ is a singular point (namely a point at which the underlying variety in real space is not a smooth manifold). In fact it is unique (up to the addition of a multiple of the hypercharge) [12] among such points in that it is a double point of both the quadratic (1e) and the cubic (1f). Particle physics cognoscenti will instantly recognize point $B$ as the combination of baryon number minus lepton number. (As we describe in [13], which studies how such singular points arise in gauge theories in general, this turns out to be no surprise.)

The utility of the point $B$ is the following. Since it is a double point of the cubic, lines through it will have similar properties to the lines through the (regular) point $C$ of the quadratic that we have already discussed: generically, a line $M$ through $B$ will intersect the cubic in at one other rational point, $X$ say, and moreover every rational point on the cubic (indeed every point in the ambient space) will lie on a line through $B$ [14].

Now let us consider the cubic and the quadratic in tandem. If $B$ were merely a regular point of the quadratic, we would face the difficulty that the point $X$ on the cubic would not normally lie on the quadratic. But because $B$ is also a double point of the quadratic, we are guaranteed that the line either lies entirely in the quadratic, or has no point in the quadratic other than $B$. On its own, this fact is not particularly useful, since it is the latter type of line which is generic [consider, e.g., the variety in $P \mathbb{Q}^{2}$ defined using coordinates $(x, y, z) \in \mathbb{Q}^{3}$ by $x y=0$, which has a double point at $(0,0,1)]$. What is needed is a construction which generically spits out lines of the former type. But this is easy: we use the original construction of rational points $R$ of the quadratic, and then consider, for each such $R$, the line $M$ joining $B$ to $R$. Generically, $R$ is distinct from $B$, in which case the line lies entirely in the quadratic (since it has a point on the quadratic, viz. $R$, which is not $B$, every point on it must be on the quadratic) and by finding the line's other intersection with the cubic, we get a new rational solution. A moment's consideration shows that all rational solutions of (1a)-(1f) can be obtained in this way.

In summary, we have the following construction, which is shown schematically in Fig. 1. Starting from a rational point on the quadratic (we take $C$, but almost any point on the quadratic distinct from $B$ would do), we construct the line $L$ joining $C$ to an arbitrary point $S$ in $P \mathbb{Q}^{13}$. This line generically hits the quadratic at a point $R$ and the line $M$ joining $R$ to the singular point $B$ (which lies in the quadratic) generically hits the cubic at a point $X$, which is a solution of (1a)-(1f). Varying the position of the point $S$ generates all solutions, so $S \in P \mathbb{Q}^{13}$ parametrizes the space of solutions.

Before delving into the nitty gritty of the parametrization, a couple of remarks are in order. One is that we must, at some point, deal with the nongeneric cases. In the construction of solutions to the quadratic, we may find that the line $L$ either lies entirely in the quadratic, or is tangent to it at $C$, meaning no further solution is

TABLE I. Sample solutions of (1a)-(1f). Point $A$ corresponds to the "Third Family Hypercharge Model" [11], while $B$ is the combination of baryon minus lepton number.

\begin{tabular}{ccccccccccccccccccc}
\hline \hline & $Q_{1}$ & $Q_{2}$ & $Q_{3}$ & $U_{1}$ & $U_{2}$ & $U_{3}$ & $D_{1}$ & $D_{2}$ & $D_{3}$ & $L_{1}$ & $L_{2}$ & $L_{3}$ & $E_{1}$ & $E_{2}$ & $E_{3}$ & $N_{1}$ & $N_{2}$ & $N_{3}$ \\
\hline$A$ & 0 & 0 & 1 & 0 & 0 & -4 & 0 & 0 & 2 & 0 & 0 & -3 & 0 & 0 & 6 & 0 & 0 & 0 \\
$B$ & 1 & 1 & 1 & -1 & -1 & -1 & -1 & -1 & -1 & -3 & -3 & -3 & 3 & 3 & 3 & 3 & 3 & 3 \\
$C$ & -1 & 0 & 1 & -1 & 0 & 1 & -1 & 0 & 1 & -1 & 0 & 1 & -1 & 0 & 1 & 0 & 0 & 0 \\
\hline \hline
\end{tabular}




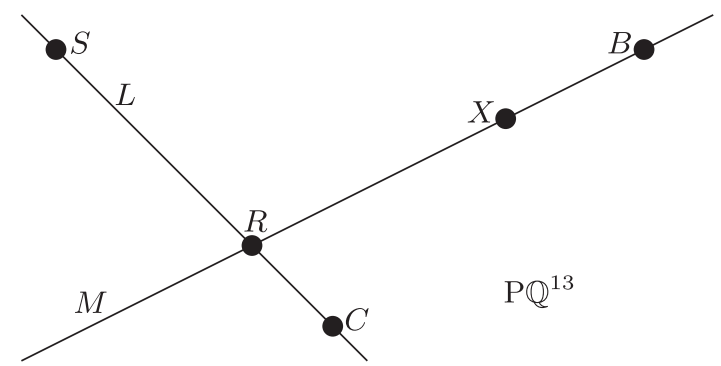

FIG. 1. Sketch of the geometric construction. $S$ is any point in the space $\mathrm{PQ}^{13}$ defined by the linear anomaly cancellation equations, $C$ is any point in $\mathrm{PQ}^{13}$ satisfying the quadratic equation and $B$ is the double point of both the quadratic and the cubic equation. $L$ is the line $C S$, which generically intersects the quadratic at $R . M$ is the line $B R$ which lies in the quadratic and generically intersects the cubic at $X$, yielding a solution to all anomaly cancellation equations.

obtained. The same situation may arise for the line $M$. As we will see, they do not cause any serious headaches. The other remark is that our parametrization of the general solution via points $S \in P \mathbb{Q}^{13}$ is clearly redundant. For example, many points $S$ will specify the same line $L$. As we shall discuss, these redundancies could easily be removed, but would result in uglier formulas.

Nitty-gritty of the solution.-Given three points $P, P^{\prime}, P^{\prime \prime}$ in $P \mathbb{Q}^{17}$ whose homogeneous coordinates are $\left(Q_{i}, U_{i}, D_{i}, L_{i}, N_{i}, E_{i}\right), \quad\left(Q_{i}^{\prime}, U_{i}^{\prime}, D_{i}^{\prime}, L_{i}^{\prime}, E_{i}^{\prime}, N_{i}^{\prime}\right)$, and $\left(Q_{i}^{\prime \prime}, U_{i}^{\prime \prime}, D_{i}^{\prime \prime}, L_{i}^{\prime \prime}, E_{i}^{\prime \prime}, N_{i}^{\prime \prime}\right)$, respectively, it will be useful to define

$$
q\left(P, P^{\prime}\right):=\sum_{i=1}^{3}\left(Q_{i} Q_{i}^{\prime}-2 U_{i} U_{i}^{\prime}+D_{i} D_{i}^{\prime}-L_{i} L_{i}^{\prime}+E_{i} E_{i}^{\prime}\right),
$$

and

$$
\begin{aligned}
c\left(P, P^{\prime}, P^{\prime \prime}\right):= & \sum_{i=1}^{3}\left(6 Q_{i} Q_{i}^{\prime} Q_{i}^{\prime \prime}+3 U_{i} U_{i}^{\prime} U_{i}^{\prime \prime}+3 D_{i} D_{i}^{\prime} D_{i}^{\prime \prime}\right. \\
& \left.+2 L_{i} L_{i}^{\prime} L_{i}^{\prime \prime}+E_{i} E_{i}^{\prime} E_{i}^{\prime \prime}+N_{i} N_{i}^{\prime} N_{i}^{\prime \prime}\right) .
\end{aligned}
$$

Now, to find the point $R$, we take a general point on the line $S C$, parametrized using homogeneous coordinates as $L=\alpha C+\beta S$, where $\alpha, \beta \in \mathbb{Q}$, and substitute into (1e), yielding

$$
\beta(2 q(C, S) \alpha+q(S, S) \beta)=0 .
$$

Cancelling the factor of $\beta$ (which appears because the point $C$ is a solution) the general solution to this equation is

$$
R=q(S, S) C-2 q(C, S) S+\delta_{q(S, S), 0} \delta_{q(C, S), 0}(a C+b S),
$$

where the Kronecker deltas (defined as $\delta_{x, y}=1$ if $x=y$ and $\delta_{x, y}=0 \forall x \neq y$ ) encode the cases where the line lies entirely within the quadratic, with $a, b \in \mathbb{Q}$ being arbitrary parameters.

To find the point $X$, we repeat the procedure, substituting the parametrization $M=\epsilon R+\gamma B$, where $\epsilon, \gamma \in \mathbb{Q}$, into the cubic (1f), yielding

$$
\epsilon^{2}(3 c(B, R, R) \gamma+c(R, R, R) \epsilon)=0 .
$$

Cancelling the factor of $\epsilon^{2}$ (which reflects the fact that $B$ is a double point of the cubic) yields

$$
\begin{aligned}
X= & c(R, R, R) B-3 c(B, R, R) R \\
& +\delta_{c(B, R, R), 0} \delta_{c(R, R, R), 0}(r B+t R),
\end{aligned}
$$

with $r, t \in \mathbb{Q}$ being arbitrary parameters.

Denoting by $S_{Q_{i}}$ the value of $Q_{i}$, etc., at the point $S$; the restriction of $S$ to the subspace $P \mathbb{Q}^{13}$ defined by the linear Eqs. (1a)-(1d) can be achieved by fixing $S_{Q_{3}}, S_{U_{3}}, S_{L_{3}}$, and $S_{E_{3}}$ by the relations

$$
\begin{aligned}
& S_{Q_{3}}=\frac{1}{2}\left(-2 S_{Q_{1}}-2 S_{Q_{2}}+\sum_{i=1}^{3}\left(S_{D_{i}}+S_{N_{i}}\right)\right), \\
& S_{U_{3}}=-\left(S_{U_{1}}+S_{U_{2}}+\sum_{i=1}^{3}\left(2 S_{D_{i}}+S_{N_{i}}\right)\right), \\
& S_{L_{3}}=-\frac{1}{2}\left(2 S_{L_{1}}+2 S_{L_{2}}+3 \sum_{i=1}^{3}\left(S_{D_{i}}+S_{N_{i}}\right)\right),
\end{aligned}
$$

$$
S_{E_{3}}=-S_{E_{1}}-S_{E_{2}}+\sum_{i=1}^{3}\left(3 S_{D_{i}}+2 S_{N_{i}}\right) \text {. }
$$

Our solution is then given in terms of the 18 parameters [16]

$$
\begin{aligned}
& S_{Q_{1}}, S_{Q_{2}}, S_{U_{1}}, S_{U_{2}}, S_{D_{1}}, S_{D_{2}}, S_{D_{3}}, S_{L_{1}}, S_{L_{2}}, S_{E_{1}}, S_{E_{2}}, S_{N_{1}}, \\
& \quad S_{N_{2}}, S_{N_{3}}, a, b, r, t \in \mathbb{Q},
\end{aligned}
$$

where the algebraic parametrization of the solution is as in (7) and $R$ is defined in (5). All that remains to write the parametrization explicitly is to substitute the charges of $B$ and $C$ from Table I. The rational solution $X$ is then given by 


$$
\begin{aligned}
& Q_{1}=\Gamma-\Sigma+\Lambda S_{Q_{1}}, \\
& Q_{2}=\Gamma+\Lambda S_{Q_{2}}, \\
& Q_{3}=\Gamma+\Sigma+\Lambda S_{Q_{3},} \\
& U_{1}=-\Gamma-\Sigma+\Lambda S_{U_{1}}, \\
& U_{2}=-\Gamma+\Lambda S_{U_{2}}, \\
& U_{3}=-\Gamma+\Sigma+\Lambda S_{U_{3}}, \\
& D_{1}=-\Gamma-\Sigma+\Lambda S_{D_{1}}, \\
& D_{2}=-\Gamma+\Lambda S_{D_{2}}, \\
& D_{3}=-\Gamma+\Sigma+\Lambda S_{D_{3}}, \\
& L_{1}=-3 \Gamma-\Sigma+\Lambda S_{L_{1}}, \\
& L_{2}=-3 \Gamma+\Lambda S_{L_{2}}, \\
& L_{3}=-3 \Gamma+\Sigma+\Lambda S_{L_{3}}, \\
& E_{1}=3 \Gamma-\Sigma+\Lambda S_{E_{1}}, \\
& E_{2}=3 \Gamma+\Lambda S_{E_{2}}, \\
& E_{3}=3 \Gamma+\Sigma+\Lambda S_{E_{3}}, \\
& N_{1}=3 \Gamma+\Lambda S_{N_{1}}, \\
& N_{2}=3 \Gamma+\Lambda S_{N_{2}}, \\
& N_{3}=3 \Gamma+\Lambda S_{N_{3}},
\end{aligned}
$$

where

$$
\begin{aligned}
\Gamma= & c(R, R, R)+r \delta_{c(B, R, R), 0} \delta_{c(R, R, R), 0}, \\
\Sigma= & \left(-3 c(B, R, R)+t \delta_{c(B, R, R), 0} \delta_{c(R, R, R), 0}\right) \\
& \times\left(q(S, S)+a \delta_{q(S, S), 0} \delta_{q(C, S), 0}\right), \\
\Lambda= & \left(-3 c(B, R, R)+t \delta_{c(B, R, R), 0} \delta_{c(R, R, R), 0}\right) \\
& \times\left(-2 q(C, S)+b \delta_{q(S, S), 0} \delta_{q(C, S), 0}\right) .
\end{aligned}
$$

This solution is provided in the ancillary directory of the arXiv preprint of this Letter in the form of a Mathematica notebook.

One way to check that the above parametrization captures all solutions is to show that it can be inverted, in the following way. For a known solution $T$ an inverse is a set of the 18 parameters (9) which return $T$ when substituted into (10). One choice of parameters which achieves this is $S=T$ and, $a=0, b=1, r=0$, and $t=1(a, b, r$, and $t$ are only needed when $T$ corresponds to one of the exceptional cases). This inverse has been successfully checked on the 21549920 solutions obtained by a scan in [8], which includes all integral solutions (up to permutations) with a maximum absolute charge up to 10 .

Closing remarks.-The general solution (10) to Eqs. (1a)-(1f) is presented for the first time. It was found by exploiting the presence of a singular point, namely the one corresponding to baryon minus lepton number, which is unique (up to the addition of a multiple of the hypercharge) in that it is a double point of both the quadratic (1e) and the cubic (1f). As such, one cannot expect the method to be of general applicability in studying anomaly cancellation in gauge theories. But it nevertheless generalizes to some situations that may be of phenomenological interest. A first generalization is to consider an arbitrary number $n$ of right-handed neutrinos (RHN). Here, it turns out that our method can be applied provided that $n$ is odd and $n \neq 1$, with the charges of the extra neutrinos at the required singular point being given by $N_{2 i}=+3, N_{2 i+1}=-3$, for $i \geq 2$. It also generalizes to an odd number of SM families with an odd number of RHN equal to or exceeding the number of families, though this is probably of lesser phenomenological interest.

Other cases require other methods, but they are not without hope. In Ref. [17], for example, a related but different method was used (following Refs. [18,19], which solved the much simpler $u(1)$ anomaly equations, i.e., without the SM part) to find a complete solution of the one SM family case (with an arbitrary number of RHN) along with a number of existence results for three families with a variety of numbers of RHN.

Our solution generalizes to real charges, corresponding to the case where the gauge group is not compact. The only change in our solution method would be changing rationals to reals everywhere, and as a consequence all parameters in (10) should be taken as real. Unlike in the one-family SM with floating real hypercharges where anomaly cancellation enforces them to be commensurate [20] here solutions exist with noncommensurate charges, for example let every SM field's charge be equal to its hypercharge and $N_{1}=\sqrt{3}$, $N_{2}=0, N_{3}=-\sqrt{3}$.

We thank other members of the Cambridge Pheno Working Group for discussions. This work has been partially supported by STFC consolidated Grants No. ST/P000681/1 and No. ST/S505316/1. B. G. is also supported by Kings College, Cambridge.

*B.C.Allanach@damtp.cam.ac.uk †ripaios@hep.phy.cam.ac.uk †jss85@cam.ac.uk

[1] Examples include models of dark matter, axions, proton stabilization, the anomalous magnetic moment of the muon, anomalies in $B$-meson decays and of fermion masses. For a review of $Z^{\prime}$ physics, see [2].

[2] P. Langacker, Rev. Mod. Phys. 81, 1199 (2009).

[3] P. Zyla et al. (Particle Data Group), Prog. Theor. Exp. Phys. 2020, 083C01 (2020).

[4] We consider here local anomalies, which require us to specify only the Lie algebra; consideration of global anomalies requires specifying the Lie group and so is more model dependent. For more details and some examples, see [5]. 
[5] J. Davighi, B. Gripaios, and N. Lohitsiri, J. High Energy Phys. 07 (2020) 232.

[6] Regarding the model as an effective field theory, it is possible that anomalies are compensated by a Wess-Zumino term. We shall ignore this possibility.

[7] A standard argument shows that vectorlike fermions make no contribution to the anomaly.

[8] B. C. Allanach, J. Davighi, and S. Melville, J. High Energy Phys. 02 (2019) 082.

[9] These arguments are standard ones in elementary number theory [10], but skeptical readers will hopefully be convinced by the explicit discussion that follows.

[10] L. Mordell, Diophantine Equations (Academic Press, New York, 1969), p. 83.

[11] B. Allanach and J. Davighi, J. High Energy Phys. 12 (2018) 075.

[12] If one were to add multiples of hypercharge to any solution, one would obtain another solution. This redundancy could be removed, resulting in the projective dimension of the variety being one fewer.

[13] B. C. Allanach, B. Gripaios, and J. Tooby-Smith (to be published).
[14] This observation goes back at least to Fermat and probably all the way to the diophantine school [15].

[15] J. Stillwell, Mathematics and its History (Springer, New York, 2010), pp. 48-49.

[16] A comment on the parameter count is in order. Generically, since we start with 18 affine parameters and have six equations, we might expect the solution to have only 12 affine parameters. The four parameters $a, b, r, t$ appear only in degenerate cases. Furthermore, one can show that it suffices to restrict to points $S$ corresponding to vectors orthogonal to both $B$ and $C$, which brings us down to the expected number. We refrain from doing so, since it complicates the (already baroque) formulas.

[17] B. C. Allanach, B. Gripaios, and J. Tooby-Smith, Phys. Rev. D 101, 075015 (2020).

[18] D. B. Costa, B. A. Dobrescu, and P. J. Fox, Phys. Rev. Lett. 123, 151601 (2019).

[19] B. C. Allanach, B. Gripaios, and J. Tooby-Smith, J. High Energy Phys. 05 (2020) 65.

[20] S. Weinberg, The Quantum Theory of Fields, Vol. 2 (Cambridge University Press, Cambridge, England, 1995). 